\title{
Life-Threatening Pulmonary Embolism: A Multidisciplinary Approach to Diagnosis and Management
}

\author{
Omar M. Lattouf, Kenneth Leeper, Heval Kelli \\ Emory University School of Medicine, Atlanta, USA \\ Email: olattou@emory.edu
}

Received August 4, 2013; revised September 4, 2013; accepted September 11, 2013

Copyright (C) 2013 Omar M. Lattouf et al. This is an open access article distributed under the Creative Commons Attribution License, which permits unrestricted use, distribution, and reproduction in any medium, provided the original work is properly cited.

\begin{abstract}
Current treatment of life threatening venous thrombo-embolism (VTE) has been based on general concepts dating to the early 1900s. In this manuscript a general overview of current diagnostic and therapeutic methods of VTE is presented along with the Emory University Affiliated Hospitals' experience of the surgical treatment of life threatening VTE. We retrospectively analyzed the data of twenty-seven consecutive patients who underwent pulmonary embolectomy on cardiopulmonary bypass from January 1998 through April 2010. Our results showed successful outcomes after urgent or emergent surgical pulmonary embolectomy. It encourages the choice of an early and aggressive surgical approach for large pulmonary emboli in hemodynamically unstable patients.
\end{abstract}

Keywords: Cardiopulmonary Bypass; Embolectomy; Pulmonary Embolism/Surgery

\section{Historical Background}

Modern day approach for the treatment of life threatening venous thrombo-embolism (VTE) dates back to over a hundred years ago and is intertwined with the history of cardio-pulmonary bypass technology and the beginning of surgical operations on the heart. Friedrich Trendelenburg in his classic 1908 paper was first to describe in lucid detail the clinical presentation of agonizing substernal pain and rapid collapse associated with sudden pulmonary embolism [1].

The recognition of the role of mechanical obstruction in the pulmonary artery by a travelling clot led to attempts to surgically remove such clots. Despite failure of early surgical attempts of pulmonary embolectomy leading to patients' death, it was Trendelenburg's student Kirschner in 1924 who was the first ever to report successful surgical embolectomy [2].

Frustrated by the high mortality rate and dismal outcome of the Trendelenburg procedure with only nine out of 142 patients treated for massive pulmonary emboli, innovative and pioneer surgeon John Gibbon initiated focused work to address this dismal outcome of the Trendelenburg procedure which led to him inventing and developing the heart lung machine and oxygenator [3]. This historic invention ushered modern era of cardiac surgery. Sharp in 1962, was the first to successfully perform pump supported pulmonary embolectomy [4].

\section{Definitions}

Life Threatening Pulmonary Embolism (LTPE) is defined as acute systemic dysfunction secondary to large veno-thrombo pulmonary embolus associated with hemodynamic instability associated with blood pressure lower than $90 \mathrm{~mm} \cdot \mathrm{Hg}$ [5].

The index criteria for meeting the definition of massive or life threatening pulmonary embolus are arterial hypotension and cardiogenic shock, manifesting as tissue hypo-perfusion, hypoxia, cool and clammy skin, oliguria and altered mental status. Arterial hypotension may be an absolute blood pressure drop to $90 \mathrm{~mm} \cdot \mathrm{Hg}$ or lower or a relative hypotension with a $40 \mathrm{~mm} \cdot \mathrm{Hg}$ drop off baseline for 15 minutes or longer [6].

Sub-massive Pulmonary Embolus is defined as imaging documented acute pulmonary embolism, without associated hypotension or shock, but with pulmonary hypertension or right ventricular dysfunction [7], as confirmed by invasive monitoring, echo-cardiography or CT scan.

\section{Epidemiology of Pulmonary Embolism}

Deep Venous Thrombosis (DVT) is a common disease process occurring annually in excess of 2 million patients in the USA. Of these, it is estimated that approximately 600,000 patients develop pulmonary emboli due to dis- 
lodgement and movement of the clot from its source of initial formation to terminate in the pulmonary artery or one of its branches. Reports indicate that upwards of 60,000 people die annually in the USA from massive PEs [8-10].

Other estimates put the annual incidence of PE-related deaths in the USA to 200,000 [11]. VTE causes more deaths than breast cancer and is the number one cause of death in post partum women [12]. It is the third most common cause of cardiovascular deaths after MI and stroke [13].

According to the International Cooperative Pulmonary Embolism Registry (ICOPER), the incidence of massive LTPE was $4.5 \%$ (108/2392 patients). In this registry the 90-day mortality rate for patients with LTPE was 52.4\%, and $14.7 \%$ for the non-LTPE. Recurrent PE were more common in the LTPE group occurring at a rate of $12.6 \%$ in the first 90 days versus $7.6 \%$ in non LTPE group. Of interest to note that in patients with massive PE thrombolytic therapy did not contribute to any significant reduction in 90-day mortality [14].

Pulmonary embolism is often a recurrent disease with a cumulative incidence of nearly $30 \%$ in eight year follow up. Post pulmonary embolism secondary pulmonary artery hypertension is reported to be at $2 \%$ in 2 years of follow-up [15].

\section{Predisposing Factors}

Patients at risk for developing acute pulmonary embolism are those who have inherited disease predisposing to hypercoagulable states such as: anti-thrombin deficiency, protein $\mathrm{C}$ deficiency, protein $\mathrm{S}$ deficiency, factor $\mathrm{V}$ Leiden, activated protein $\mathrm{C}$ resistance without factor $\mathrm{V}$ Leiden, prothrombin gene mutation, dysfibrinogenemia and plasminogen deficiency. The following acquired factors: reduced mobility, advanced age, cancer, acute medical illness, major surgery, trauma, spinal cord injury, pregnancy and postpartum period, polycythemia vera, antiphospholipid antibody syndrome, oral contraceptives, hormone-replacement therapy, Heparins exposure, chemotherapy, obesity, central venous catheterization, immobilizer or cast have been associated with increased risks for veno-thrombo embolism [16].

\section{Pathophysiology of Pulmonary Embolism}

The mechanical obstruction caused by large obstructive emboli compromising or occluding outflow from the RV and inflow into the pulmonary circulation and thus limiting pulmonary circulation and reducing return of oxygenated blood into the left heart is recognized as the fundamental pathophysiology of LTPE. In addition, humoral factors such as serotonin release from platelets, thrombin release from plasma, and histamine release from ischemic lung tissue contribute to vasospasm in the pulmonary micro circulation leading to increased pulmonary vascular resistance, exacerbation and worsening pulmonary hypertension. The increased right ventricular after-load causes right ventricular dilatation, hypokinesis, and tricuspid regurgitation with annular dilatation of the tricuspid valve, and ultimately severe right ventricularstrainand failure. The hemodynamic response to PE is determined by the size of the embolus and the patient's underlying cardiopulmonary status [17].

RV dysfunction (dilation and hypokinesis) resulting from acute pressure overload is capable of initiating a vicious cycle of increased myocardial oxygen demand, myocardial ischemia (or infarction), left ventricular preload reduction, and, ultimately, inability to maintain the cardiac index and arterial pressure [18]. In the acute phase of massive PEs, right ventricular tension rise leads to dilatation, dysfunction, and ischemia, failure and death.

The lower extremity veins are usually the site for thrombus formation and source for clot migration to the lungs [16]. Veno thrombo emboli have been reported from deep vein thrombi developing in upper extremity veins. Irrespective of source of thrombi, and more importantly their size may cause sudden onset of cascading events leading to acute illness manifesting with shortness of breath, chest pain, pleuritic pain, hemoptysis, anxiety, tachycardia, hypoxia, variable degree of right heart strain or failure, hypotension, cardiogenic shock or death.

Right ventricular strain is often noted on ECG with incomplete or complete right bundle-branch block, Twave inversions in the anterior precordium, and an $\mathrm{S}$ wave in lead I and a Q-wave and T-wave inversion in lead III (S1Q3T3). In young, previously healthy patients, the ECG may be entirely normal [19].

The wide spread availability of CT scanners has made CT angiograms a commonly used emergency room modality for evaluation of sudden chest pain to rule out aortic dissections and pulmonary emboli. This has led to increased rate of detection of $\mathrm{PE}$ and has reduced the reliance on ventilation perfusion scans, unless reasons exist to avoid contrast dye patient-exposure. The exceedingly high negative predictive value (99\%) of CTA in the evaluation of suspected PEs has made CTA the procedure of choice to rule out pulmonary emboli [20].

In acute $\mathrm{PE}$, it has been reported that RV enlargement to $90 \%$ of the LV on CT scan is a strong predictor of 90day mortality with a hazard ratio of 3.36 [21].

\section{Clinical Presentation}

There is no uniform clinical presentation for pulmonary embolism. Presentation may vary widely between one patient and another. In some, the presentation may be 
limited to the most common presenting factor; dyspnea while in others pulmonary emboli may present with dyspnea, pleuritic pain and cyanosis indicating a significant embolus. Small peripherally located PEs may be associated with pleuritic pain, cough, hemoptysis and dyspnea [19].

On physical examination, tachypnea and anxiety are the two most common sign of pulmonary emboli. Of note however is that the hemodynamic sequel of PE; hypotension, cardiogenic shock, or cardiac arrest, may be more related to the cardio-pulmonary reserve of the individual patient than the absolute size of the PE [19].

In massive PEs, if condition is not promptly reversed and pulmonary flow is not resumed, this sequence of events may lead to fatal cardiogenic shock. Most PErelated deaths are due to refractory cardiac (rather than respiratory) failure. Overt RV failure caused by mechanical obstruction of blood out-flow from the right ventricle and compounded by secondary pulmonary circulation vasospasm lead to poor filling of the left ventricle with refractory arterial hypotension and shock [7].

\section{Laboratory Findings}

Being a standard and commonly used laboratory test for evaluation of chest related problem, the ECG once done may reveal signs of RV strain, including incomplete or complete right bundle-branch block, S wave in lead I and a Q-wave and T-wave inversion in lead III (S1Q3T3). A normal ECG should not be used as indication to rule out PE. Completely normal ECG may occur in young, healthy patients with no prior history of cardiac diseases in the presence of pulmonary embolus.

Routine CXR is rarely, if ever anymore, relied upon to diagnose PE. More often the CXR is normal. Historically however several radiographic signs have been used to diagnose PEs such as: Westermark sign caused by focal oligemia in the lung field, Hampton's hump-a peripheral wedge shaped opacity, or Palla's sign indicating an enlarged right descending pulmonary artery [22].

Chest CT angiography has become the main laboratory diagnostic test to confirm or rule out pulmonary emboli. The rapid advancements in CTA technology have allowed rapid detection of large saddle emboli, branch pulmonary artery lesions or distal sub-segmental emboli. CT angiography has practically replaced all other modalities in diagnosing PEs or ruling out PE as cause of disease in question [22]. Contrast-enhanced chest CT is increasingly used as the first-line PE imaging test and is available 24 hours daily at most institutions [23].

For patients with contrast dye allergy or those with renal insufficiency, non dye contrast studies such as ventilation perfusion scans remain valuable. Trans-thoracic echoes and trans-esophageal echoes are PE diagnosti- cally insensitive. However they are valuable of assessing RV function and play an important role in risk stratification of patients with proven pulmonary embolism [19].

\section{Therapeutic Strategies}

\subsection{Anti-Coagulant and Thrombolytic Therapy}

Kucher at al in a study on 2392 patients from the International Cooperative Pulmonary Embolism Registry (ICOPER), reported that 108 (4.5\%) had massive PEs, and 2284 (94.5) had non-massive PE. Ninety-day mortality rates were $52.4 \%$ and $14.7 \%$ respectively.

In the massive PE group, heparin plus thrombolytic therapy did not provided a significant survival advantage over those who received Heparin only (90-day mortality of $46 \%$ vs. $55 \%$ ). Bleeding complications were much more frequent in the thrombolytic sub-group; $24 \%$ vs $15 \%$ in non thrombolytic sub-group. Recurrent PEs occurred with equal frequency of $12 \%$ in both sub-groups. IVC filters, however, was associated with reduction of 90 day mortality (HR 0.12 p: <0.002) [14].

A meta analysis by Thabut et al. on 461 patients from nine different trials with documented PEs, thrombolytic therapy had no significant advantage over intravenous heparin in regards to mortality or recurrence of $\mathrm{PE}$ but was associated with an increased risk of major hemorrhage, irrespective of the formulation of thrombolytic agent, clinical severity of PE or extent of vascular obstruction [24].

In addition to the short term morbidity and mortality associated with pulmonary embolism, the long term effects of PE remain significant be they due to the post thrombotic syndrome with secondary venous insufficiency in the lower extremities [9] or the long term effects of chronic pulmonary hypertension and its impact on cardio respiratory reserves and reduced longevity [15].

\subsection{Surgical Therapy}

In the minds of many internists, the indications and value of surgical pulmonary embolectomy remains poorly understood or at best considered a high-risk procedure or court of last resort offered only to patients in extremis. Perhaps this misconception is due to reliance on outdated data of the 1960s when mortality rate associated with surgical pulmonary embolectomy was in excess of 50\% [25]. Further review of the literature reveals impressive gradual reduction in mortality rate to $25 \%$ in the 1990 [26] and further down to 6\% in the current era [27].

Others have corroborated these current impressive survival rates with similar low operative $(6 \%)$ mortality in patients with massive pulmonary emboli. Modern day surgical embolectomy is associated with long term bene- 
fit advantage with a relatively low all cause mortality at 1 and 3 years of $14 \%$ and $17 \%$ [28,29].

The improved and encouraging survival outcome in short and intermediate term in the massive PE patients has encouraged aggressive surgical approach to be offered to the sub-massive patient population. Digonnet has reported $100 \%$ operative and one-year survival in the surgical treatment for sub-massive PEs. These data support broader application of surgical embolectomy as primary treatment for patients with massive or sub-massive pulmonary emboli [29].

\section{Brief Summary of the Emory Experience and Sample Case Presentations}

From January 1998 through April 2010 twenty-sevenconsecutive patients underwent pulmonary embolectomy on cardiopulmonary bypass. Retrospective data review revealed that mean age was 55.6 years $( \pm 15.4)$, with $55.6 \%$ females, 6 (22\%) with diabetes mellitus, 6 (22\%) smokers, 3 (11\%) immune-suppressed, 4 (14.8\%) with chronic lung disease, 5 (18.5\%) presented with angina, 10 (37\%) with MI, 11 (40.7\%) in heart failure, 19 (70\%) history systemic arterial hypertension and $17(63 \%)$, presented in critical hemodynamics requiring immediate surgical intervention (Table 1). Thrombus extraction from the main pulmonary artery, secondary and tertiary branches were uniformly successful. There were no 30day mortalities, nor postoperative MI, stroke, pneumonia, renal failure, sternal wound infection, or retroperitoneal/ intra-cerebral bleeding. Two late deaths at 2 and 10 months after discharge were unrelated to the pulmonary embolism or embolectomy procedure (Graph 1).

\subsection{Case 1 (Figures 1(a) and (b))}

Fifty-one-year-old male, presented with acute chest pain, ruled out for acute coronary syndrome or coronary artery

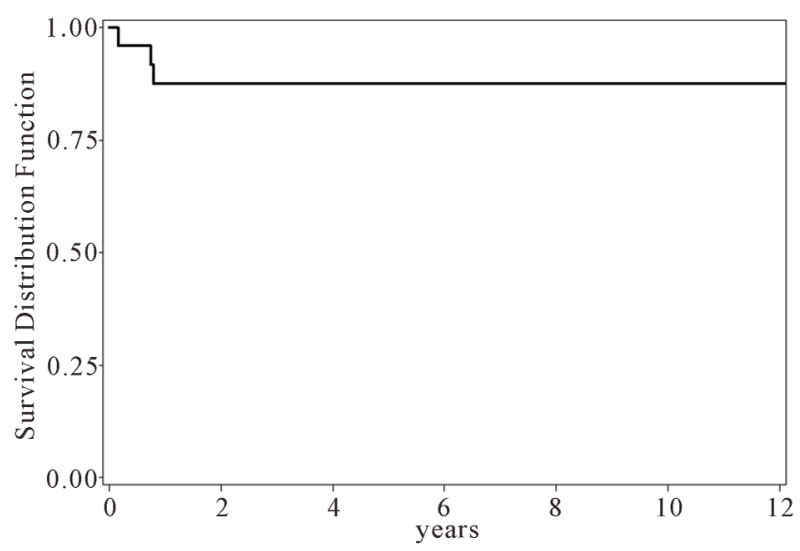

Graph 1. Long term survival in post surgical embolectomy patients.
Table 1. Characteristics.

\begin{tabular}{|c|c|}
\hline Characteristic & Distribution $\mathrm{N}=27$ \\
\hline \multicolumn{2}{|c|}{ PREOPERATIVE VARIABLES } \\
\hline Patient Age, mean (SD) & $55.6(15.4)$ \\
\hline Female Gender, n (\%) & $15(55.6)$ \\
\hline Caucasian Race, n (\%) & $17(63.0)$ \\
\hline Weight in kg, mean (SD) & $91.4(20.2)$ \\
\hline Height in cm, mean (SD) & $170.5(10.4)$ \\
\hline BMI, mean (SD) & $31.5(7.2)$ \\
\hline Last Creatinine Level, mean (SD) & $1.18(0.46)$ \\
\hline Redo, n (\%) & $2(7.4)$ \\
\hline Smoker, n (\%) & $6(22.2)$ \\
\hline Cerebrovascular Disease, n (\%) & $2(7.4)$ \\
\hline Chronic Lung Disease, n (\%) & $4(14.8)$ \\
\hline Diabetes, n (\%) & $6(22.2)$ \\
\hline Family History of CAD, n (\%) & $3(11.1)$ \\
\hline Hypertension, n (\%) & $19(70.4)$ \\
\hline Immunocompromised, n (\%) & $3(11.1)$ \\
\hline Renal Failure, n (\%) & $1(3.7)$ \\
\hline Dialysis, n (\%) & $1(3.7)$ \\
\hline Angina, n (\%) & $5(18.5)$ \\
\hline Recent Heart Failure, n (\%) & $11(40.7)$ \\
\hline MI, n (\%) & $10(37.0)$ \\
\hline Emergent Status, n (\%) & $17(63.0)$ \\
\hline Ejection Fraction, n (\%) & $59.4(6.8)$ \\
\hline \multicolumn{2}{|c|}{ INTRA-OPERATIVE VARIABLES } \\
\hline CPB Time, mean (SD) & $65.5(43.3)$ \\
\hline Concomitant CAB, mean (SD) & $2(7.4)$ \\
\hline \multicolumn{2}{|c|}{ POST-OPERATIVE VARIABLES } \\
\hline LOS, mean (SD) & $11.0(8.2)$ \\
\hline ICU Hours, mean (SD) & 102 (132), Median = 57 \\
\hline Venthours, mean (SD) & 58.7 (124.1), Median = 16.5 \\
\hline Blood Products, n (\%) & 15 (55.6) \\
\hline Stroke, n (\%) & $0(0.0)$ \\
\hline MI, n (\%) & $0(0.0)$ \\
\hline Renal Failure, n (\%) & $2(7.4)$ \\
\hline Pneumonia, n (\%) & $4(14.8)$ \\
\hline Prolonged Ventilation, n (\%) & $9(33.3)$ \\
\hline 30-Day Mortality, n (\%) & $0(0.0)$ \\
\hline 1-Year Survival & $87.7 \%$ \\
\hline 3-Year Survival & $87.7 \%$ \\
\hline 5-Year Survival & $87.7 \%$ \\
\hline 10-Year Survival & $87.7 \%$ \\
\hline
\end{tabular}




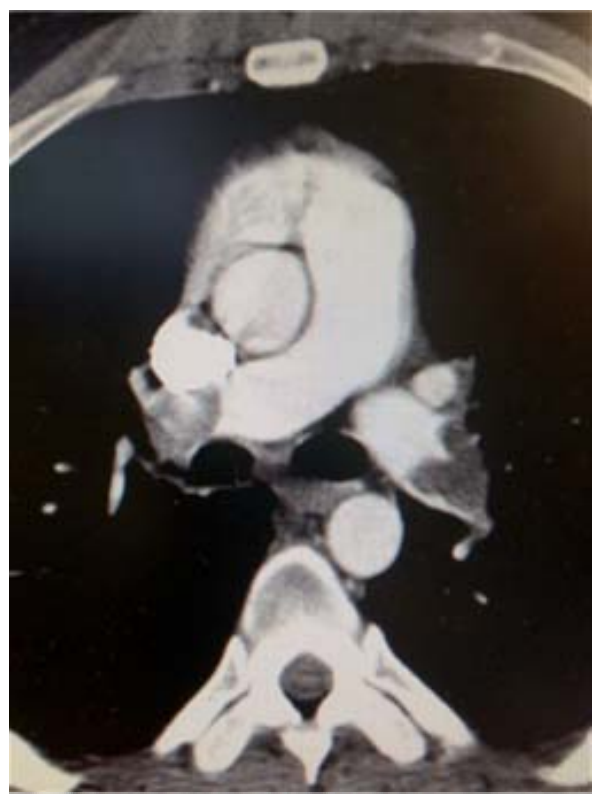

(a)

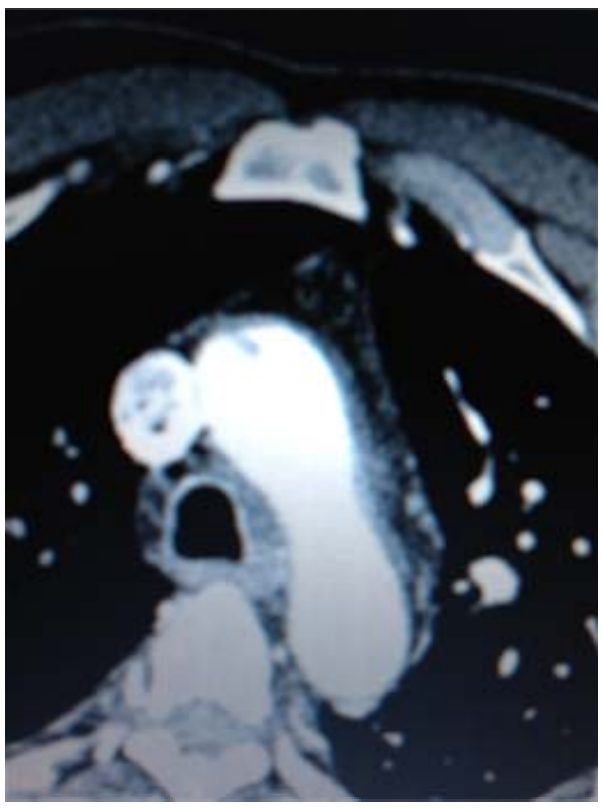

(b)

Figure 1. (a) Large bilateral PEs; (b) Ulcerated distal ascending aortic plaque with clot and superior vena cava clot.

disease by cardiac catheterization. He continued to have dyspnea at rest, thus an echocardiogram confirmed an image of strained right ventricle and right atrium and the inter-ventricular septum bowed to left. Suspension for pulmonary embolism was confirmed with CT angiogram revealing massive bilateral pulmonary emboli.

On cardio-pulmonary bypass and circulatory arrest, uneventful extraction of clots from superior vena cava, right and left pulmonary arteries, and distal ascending aorta was done.

\subsection{Case 2 (Figure 2)}

A seventy-seven-year-old female developed new onset right leg swelling after hospitalization for severe achalasia and undergoing esophageal dilatation. During her in-hospital course, she had a sudden episode of dyspnea, hypoxia and hypotension. Echo revealed severe right ventricular strain along with "clots-in-transit" in the inferior vena cava and right atrium. Emergency surgical intervention with removal of clots from right atrium, inferior vena cava, right and left pulmonary arteries along with closure of patent foramen ovale and insertion of inferior vena cava umbrella resulted in resolution of symptoms and favorable outcome.

\subsection{Case 3 (Figures 3(a) and (b))}

A sixty-year-old male with history of diabetes mellitus, prior strokes, deep venous thrombosis, presented with hypotension, acute cardiac and pulmonary decompensation with peripheral oxygen saturation in $60 \mathrm{~s}$, echo evidence of right ventricular distension and serum troponin at 0.05. Emergent successful bilateral pulmonary embolectomy on CPB and insertion of IVC filter were carried out.

\subsection{Case 4 (Figures 4(a) and (b))}

A 21-year-old female patient, 26 weeks gravid with twins, presented to an outside hospital with back pain and shortness of breath. Past medical history significant for old motor vehicle related paraplegia.

Successful C-section/delivery of healthy twins, followed immediately with median sternotomy, extraction of thrombus from PFO, suture closure of PFO and insertion of IVC filter.

\section{Conclusion}

This review of the literature indicates that although

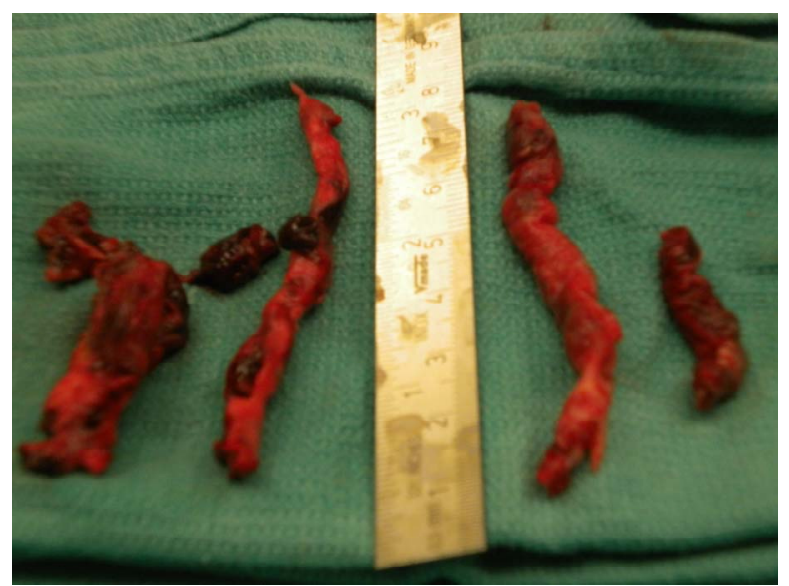

Figure 2. Multiple large extracted pulmonary embol. 


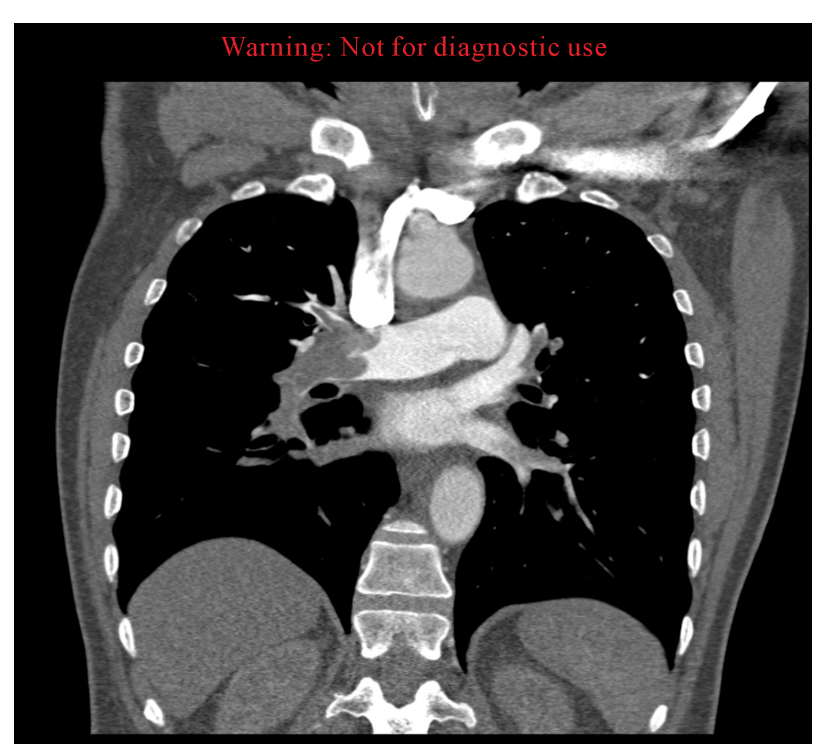

(a)

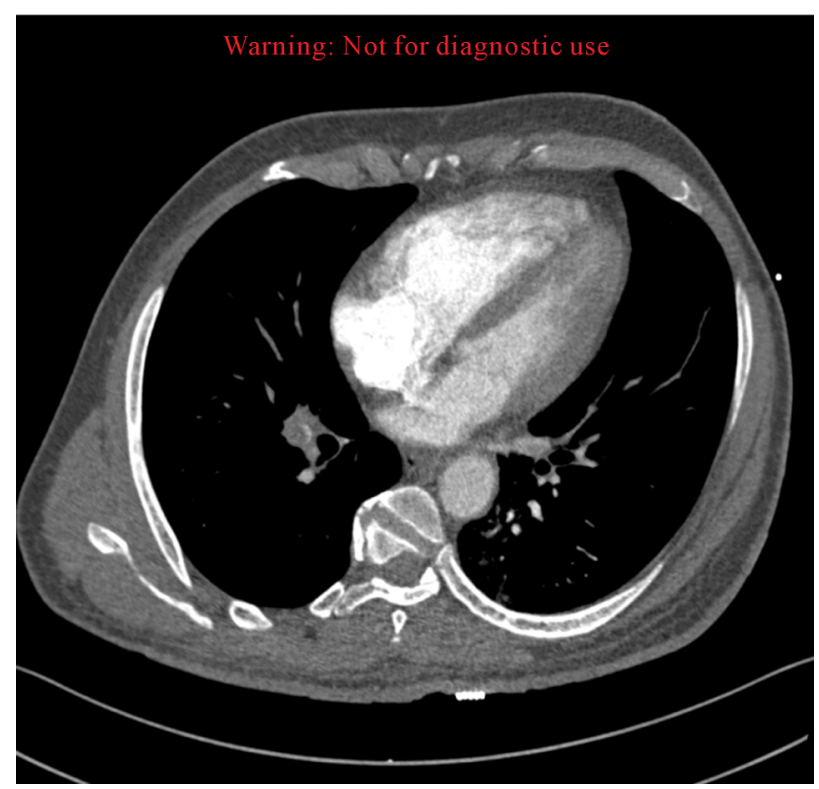

(b)

Figure 3. (a) Total occlusion of right pulmonary artery; (b) CTA demonstrating distended right ventricle.

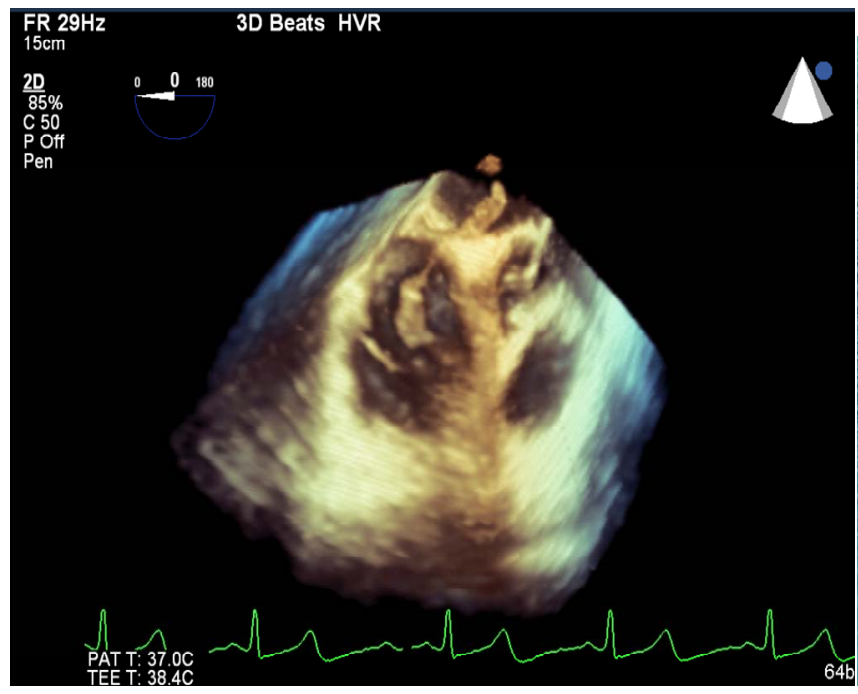

(a)

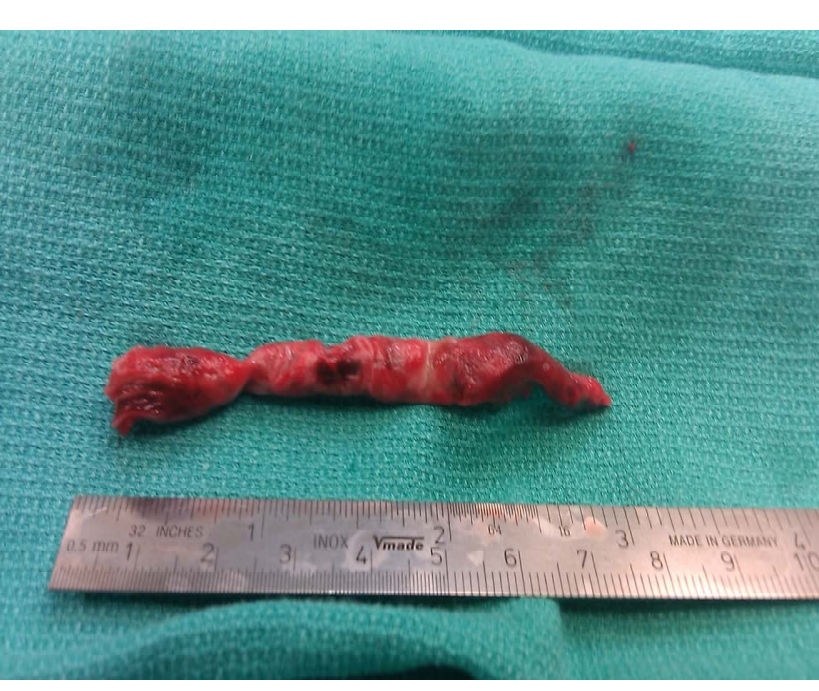

(b)

Figure 4. (a) Thrombus straddling inter-atrial septum; (b) Extracted embolus from patent foramen ovale.

acute pulmonary embolism remains a significant public health threat as the third most common cardiovascular cause of death, the current guidelines consider thrombolysis the primary therapy despite its high frequency of retroperitoneal and intracranial bleeding (15\% - 24\%) and 90-day mortality (46\% - 55\%). Additionally often, surgical referrals are often delayed until clinical extremis occurs. Our results presented here of excellent clinical outcomes after urgent or emergent surgical pulmonary embolectomy support an aggressive surgical approach as primary treatment for large pulmonary emboli in patients with hemodynamic instability, echo-documented right ventricular strain or presence of secondary cardiac clots.

\section{REFERENCES}

[1] F. Trendelenburg, "Operative Management of Pulmonary Emboli,” Arch Klin Chir, Vol. 86, 1908, pp. 686-700.

[2] M. Imielinski, et al., "Common Variants at Five New Loci Associated with Early-Onset Inflammatory Bowel Disease," Nature Genetics, Vol. 41, No. 12, 2009, pp. 1335-1340. http://dx.doi.org/10.1038/ng.489

[3] J. Gibbon, "Artificial Maintenance of Circulation during Experimental Occlusion of Pulmonary Artery," Archives of Surgery, Vol. 34, No. 6, 1937, pp. 1105-1131. 
[4] E. H. Sharp, "Pulmonary Embolectomy: Successful ReMoval of a Massive Pulmonary Embolus with the Support of Cardiopulmonary Bypass, Case Report," Annals of Surgery, Vol. 156, No. 1, 1962, pp. 1-4. http://dx.doi.org/10.1097/00000658-196207000-00001

[5] S. Z. Goldhaber, "Pulmonary Embolism," Lancet, Vol. 363, No. 9417, 2004, pp. 1295-305. http://dx.doi.org/10.1016/S0140-6736(04)16004-2

[6] W. Kasper, et al., "Management Strategies and DetermiNants of Outcome in Acute Major Pulmonary Embolism: Results of a Multicenter Registry," Journal of the American College of Cardiology, Vol. 30, No. 5, 1997, pp. $1165-1171$ http://dx.doi.org/10.1016/S0735-1097(97)00319-7

[7] S. Konstantinides, et al., "Heparin plus Alteplase Compared with Heparin Alone in Patients with Submassive Pulmonary Embolism," The New England Journal of Medicine, Vol. 347, No. 15, 2002, pp. 1143-1150. http://dx.doi.org/10.1056/NEJMoa021274

[8] I. Rubinstein, D. Murray and V. Hoffstein, "Fatal Pulmonary Emboli in Hospitalized Patients. An Autopsy Study," Archives of Internal Medicine, Vol. 148, No. 6, 1988, pp. 1425-1426.

http://dx.doi.org/10.1001/archinte.1988.00380060189033

[9] J. E. Dalen and J. S. Alpert, "Natural History of Pulmonary Embolism," Progress in Cardiovascular Diseases, Vol. 17, No. 4, 1975, pp. 259-270.

http://dx.doi.org/10.1016/S0033-0620(75)80017-X

[10] W. R. Bell and T. L. Simon, "Current Status of Pulmonary Thromboembolic Disease: Pathophysiology, Diagnosis, Prevention, and Treatment," American Heart Journal, Vol. 103, No. 2, 1982, pp. 239-262. http://dx.doi.org/10.1016/0002-8703(82)90498-7

[11] J. B. Dimick, et al., "Hospital Costs Associated with Surgical Complications: A Report from the Private-Sector National Surgical Quality Improvement Program,” Journal of the American College of Surgeons, Vol. 199, No. 4, 2004, pp. 531-537. http://dx.doi.org/10.1016/j.jamcollsurg.2004.05.276

[12] A. M. Kaunitz, et al., "Causes of Maternal Mortality in the United States," Obstetrics and Gynecology, Vol. 65, No. 5, 1985, pp. 605-612.

[13] F. A. Anderson Jr., et al., “A Population-Based Perspective of the Hospital Incidence and Case-Fatality Rates of Deep Vein Thrombosis and Pulmonary Embolism. The Worcester DVT Study," Archives of Internal Medicine, Vol. 151, No. 5, 1991, pp. 933-938.

http://dx.doi.org/10.1001/archinte.1991.00400050081016

[14] N. Kucher, et al., "Massive Pulmonary Embolism," Circulation, Vol. 113, No. 4, 2006, pp. 577-582. http://dx.doi.org/10.1161/CIRCULATIONAHA.105.5925 $\underline{92}$

[15] V. Pengo, et al., "Incidence of Chronic Thromboembolic Pulmonary Hypertension after Pulmonary Embolism," The New England Journal of Medicine, Vol. 350, No. 22, 2004, pp. 2257-2264.

http://dx.doi.org/10.1056/NEJMoa032274

[16] V. F. Tapson, “Acute Pulmonary Embolism,” The New
England Journal of Medicine, Vol. 358, No. 10, 2008, pp. 1037-1052. http://dx.doi.org/10.1056/NEJMra072753

[17] S. Z. Goldhaber and C. G. Elliott, “Acute Pulmonary EmBolism: Part I: Epidemiology, Pathophysiology, and Diagnosis," Circulation, Vol. 108, No. 22, 2003, pp. 27262729.

http://dx.doi.org/10.1161/01.CIR.0000097829.89204.0C

[18] J. C. Lualdi and S. Z. Goldhaber, "Right Ventricular DysFunction after Acute Pulmonary Embolism: PathophysiOlogic Factors, Detection, and Therapeutic Implications," American Heart Journal, Vol. 130, No. 6, 1995, pp. 1276-1282. http://dx.doi.org/10.1016/0002-8703(95)90155-8

[19] G. Piazza and S. Z. Goldhaber, "Acute Pulmonary Embolism: Part I: Epidemiology and Diagnosis,” Circulation, Vol. 114, No. 2, 2006, pp. e28-e32. http://dx.doi.org/10.1161/CIRCULATIONAHA.106.6208 $\underline{72}$

[20] U. J. Schoepf, et al., "Subsegmental Pulmonary Emboli: Improved Detection with Thin-Collimation Multi-Detector Row Spiral CT,” Radiology, Vol. 222, No. 2, 2002, pp. 483-490.

http://dx.doi.org/10.1161/CIRCULATIONAHA.106.6208 72

[21] U. J. Schoepf and P. Costello, "CT Angiography for Diagnosis of Pulmonary Embolism: State of the Art," Radiology, Vol. 230, No. 2, 2004, pp. 329-337. http://dx.doi.org/10.1148/radiol.2302021489

[22] P. D. Stein, F. Kayali and R. E. Olson, “Trends in the Use of Diagnostic Imaging in Patients Hospitalized with Acute Pulmonary Embolism," The American Journal of Cardiology, Vol. 93, No. 10, 2004, pp. 1316-1317. http://dx.doi.org/10.1016/j.amjcard.2004.02.022

[23] U. J. Schoepf, et al., "Right Ventricular Enlargement on Chest Computed Tomography: A Predictor of Early Death in Acute Pulmonary Embolism," Circulation, Vol. 110, No. 20, 2004, pp. 3276-3280. http://dx.doi.org/10.1161/01.CIR.0000147612.59751.4C

[24] G. Thabut, et al., "Thrombolytic Therapy of Pulmonary Embolism: A Meta-Analysis,” Journal of the American College of Cardiology, Vol. 40, No. 9, 2002, pp. 16601667. http://dx.doi.org/10.1016/S0735-1097(02)02381-1

[25] F. S. Cross and A. Mowlem, "A Survey of the Current Status of Pulmonary Embolectomy for Massive Pulmonary Embolism,” Circulation, Vol. 35, Suppl. 4, 1967, pp. I86-I91.

[26] P. Stulz, et al., "Decision Making in the Surgical Treatment of Massive Pulmonary Embolism,” European Journal of Cardio-Thoracic Surgery: Official Journal of the European Association for Cardio-Thoracic Surgery, Vol. 8, No. 4, 1994, pp. 188-193. http://dx.doi.org/10.1016/1010-7940(94)90113-9

[27] K. Yalamanchili, et al., "Open Pulmonary Embolectomy for Treatment of Major Pulmonary Embolism,” The Annals of Thoracic Surgery, Vol. 77, No. 3, 2004, pp. 819-823. http://dx.doi.org/10.1016/j.athoracsur.2003.08.008

[28] M. Leacche, et al., "Modern Surgical Treatment of Mas- 
sive Pulmonary Embolism: Results in 47 Consecutive Patients after Rapid Diagnosis and Aggressive Surgical Approach,” The Journal of Thoracic and Cardiovascular Sugery, Vol. 129, No. 5, 2005, pp. 1018-1023.

http://dx.doi.org/10.1016/j.jtcvs.2004.10.023
[29] A. Digonnet, et al., “Acute Pulmonary Embolism: A CurRent Surgical Approach,” Interactive Cardiovascular and Thoracic Surgery, Vol. 6, No. 1, 2007, pp. 27-29. http://dx.doi.org/10.1510/icvts.2006.141366 